\title{
Nonlinear Interactions between Free Electrons
}

\section{and Nanographenes}

\author{
Joel D. Cox ${ }^{\dagger, \dagger}$ and F. Javier García de Abajo*,,$\S$ \\ $\dagger$ Center for Nano Optics, University of Southern Denmark, Campusvej 55, DK-5230 \\ Odense $M$, Denmark \\ $\ddagger$ Danish Institute for Advanced Study, University of Southern Denmark, Campusvej 55, \\ DK-5230 Odense M, Denmark \\ IICFO-Institut de Ciencies Fotoniques, The Barcelona Institute of Science and \\ Technology, 08860 Castelldefels (Barcelona), Spain \\ §ICREA-Institució Catalana de Recerca i Estudis Avançats, Passeig Lluís Companys 23, \\ 08010 Barcelona, Spain \\ E-mail: javier.garciadeabajo@nanophotonics.es
}

\begin{abstract}
Free electrons act as a source of highly confined, spectrally broad optical fields that are widely used to map photonic modes with nanometer/millielectronvolt space/energy resolution through currently available electron energy-loss and cathodoluminescence spectroscopies. These techniques are understood as probes of the linear optical response, while nonlinear dynamics has escaped observation with similar degree of spatial detail, despite the strong enhancement of the electron evanescent field with decreasing electron energy. Here, we show that the field accompanying low-energy electrons can trigger anharmonic response in strongly nonlinear
\end{abstract}


materials. Specifically, through realistic quantum-mechanical simulations, we find that the interaction between $\lesssim 100 \mathrm{eV}$ electrons and plasmons in graphene nanostructures gives rise to substantial optical nonlinearities that are discernable as saturation and spectral shifts in the plasmonic features revealed in the cathodoluminescence emission and electron energy-loss spectra. Our results support the use of low-energy electron-beam spectroscopies for the exploration of nonlinear optical processes in nanostructures.

Keywords: nonlinear optics, electron beams, graphene plasmons, electron microscopy, nanographenes, cathodoluminescence

Optical spectroscopies rely on the response of the sample to the electromagnetic field of an external light source. When combined with far-field microscopy, the spatial resolution of these techniques is limited by diffraction to roughly half the light wavelength, ${ }^{1}$ while the use of tips in scanning near-field optical setups permits imaging features with sizes down to tens of nanometers. ${ }^{2}$ A substantial gain in spatial resolution can be achieved if the external field is supplied by electron beams (e-beams), which can be currently focused down to sub-Ångstrom spots, where spectral analysis of the energy losses experienced by the electrons $^{3-5}$ or the cathodoluminescence (CL) light emission resulting from their interaction with the sample ${ }^{6}$ allow us to identify optical excitations with millielectronvolt energy resolution. In an intuitive picture, the passage of an electron produces a transient evanescent electric field that can be regarded as a broad optical pulse capable of exciting the sample. In CL spectroscopy, an optical monochromator separates different frequency components of the scattered far-field (i.e., different emitted photon energies) resulting from that interaction, while in electron energy-loss spectroscopy (EELS) an electron analyzer is used to resolve the excitation frequencies as spectral features associated with energy losses experienced by the transmitted electrons. These techniques have been extensively applied to study plasmons in nanostructures, ${ }^{7-12}$ optical modes in photonic crystals, ${ }^{13-15}$ and more recently, localized phonon polaritons in the mid-infrared spectral range. ${ }^{3-5}$ The interaction of e-beams with 
engineered photonic structures has been also explored as a mechanism for integrated light sources. ${ }^{16,17}$ Adding to the unprecedented combination of space and energy resolution enabled by electron beams, ultrafast temporal resolution has been achieved through the use of ultrashort electron pulses emitted from a cathode under femtosecond laser pulse irradiation. ${ }^{18-20}$

Because the excitation yield of optical modes by e-beams is generally found to be small (e.g., $<10^{-4}$ per electron at typical beam energies $\left.\sim 100 \mathrm{keV}\right)$, EELS and CL are commonly regarded as probes of the linear optical response. Nevertheless, the amplitude of the evanescent field provided by a moving electron at a specific frequency (i.e., the spectral component of that field in the frequency range of the sampled mode) scales as $\sim 1 / v$ at low velocity $v \ll c,{ }^{6}$ thus resulting in $\mathrm{a} \sim 1 / v^{2}$ dependence of the excitation yield. In fact, for $<100 \mathrm{eV}$ electrons and strong plasmonic optical modes, the interaction has been theoretically shown to reach unity order. ${ }^{21}$ We thus expect that low-energy e-beams can trigger an anharmonic response in strongly nonlinear nanostructures such as graphene nanoislands, where a departure from the linear regime should be already observable at the level of a single plasmon excitation. ${ }^{22}$

Here, we demonstrate through realistic quantum-mechanical simulations that low-energy e-beams can indeed trigger nonlinear optical response in graphene nanoislands, giving rise to saturation and sizeable frequency shifts of the peaks in CL emission and energy loss spectra associated with the excitation of plasmons in the sample. We base these results on time-domain simulations of the electron-graphene interaction, with the latter described in a self-consistent field approximation that incorporates nonlinear processes at all orders, and the electron introduced as the external field produced by a moving point charge. Nonlinear effects are revealed by studying the dependence of CL and energy loss spectra on the charge of the probe $q$, which shows a clear departure from the linear regime (i.e., quadratic dependence on $q$ ) already for $q=-e$ (one electron). In a practical scenario, we find that nonlinear effects can be probed by studying the dependence of the emission or energy loss spectra on e-beam 
energy and lateral position, where spectral shifts as large as the plasmon linewidth should be feasible under optimal conditions. The present study supports the potential of low-energy electrons to explore the nonlinear optical response of nanostructures with unprecedented spatial resolution.
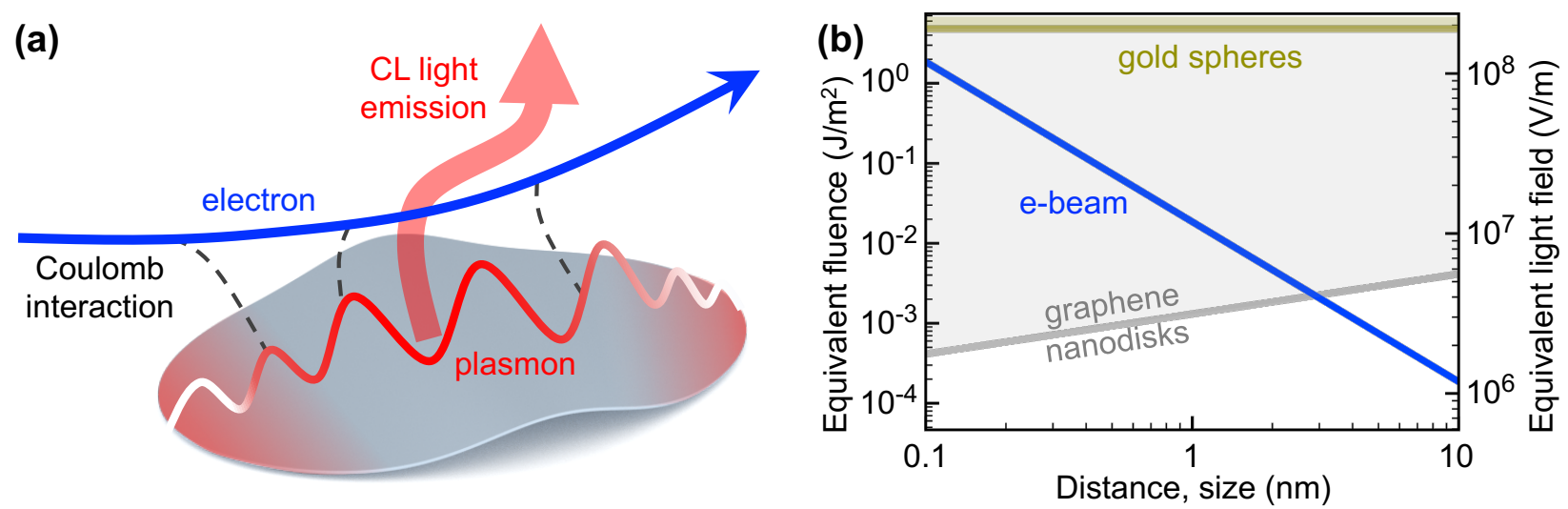

Figure 1: Nonlinear optics with electron beams. (a) A free electron passing near a nanostructure can interact multiple times via its evanescent Coulomb field with one of the sample plasmons, triggering nonlinear optical response that in turn leaves a signature in the resulting cathodoluminescence (CL) light emission. (b) The strength of the field produced by the electron depends on distance to its trajectory and has an effect on a sample resonance of lifetime $\tau$ similar to a spectrally narrow light pulse with an equivalent fluence $\mathcal{F}^{\text {eff }} \approx e^{2} c /\left(2 \pi v^{2} R^{2} \tau\right)$ (left scale) and electric field strength $E=(2 e / v R) / \sqrt{\tau \Delta}$ (right scale), as shown in this plot for $25 \mathrm{eV}$ electron energy $(v \approx c / 100), \hbar \tau^{-1}=10 \mathrm{meV}(\tau \approx 66 \mathrm{fs})$, and $\Delta=100 \mathrm{fs}$ light pulse duration. Estimates of the onsets of nonlinear response in self-standing gold spheres and silicon-supported graphene disks are shown for comparison as a function of their size (see main text).

Equivalent Optical Pulse Fluence of a Free Electron. Electrons moving in vacuum produce an evanescent electromagnetic field that can interact with the optical modes of a sample, giving rise to energy losses and emission of radiation ${ }^{6}$ (Figure 1a). This is the basis of the EELS and CL techniques, the spectra of which are conveniently studied by time-Fourier transforming the electric field produced by the electron, $\mathbf{E}^{\operatorname{ext}}(\mathbf{r}, t)=\int(d \omega / 2 \pi) \mathrm{e}^{-\mathrm{i} \omega t} \mathbf{E}^{\mathrm{ext}}(\mathbf{r}, \omega)$. For constant nonrelativistic velocity $v \ll c$, the frequency-space electric field intensity reduces to $^{6}\left|\mathbf{E}^{\mathrm{ext}}(\mathbf{r}, \omega)\right|^{2}=\left(2 e \omega / v^{2}\right)^{2} K_{m}^{2}(\omega R / v)$ as a function of distance $R$ normal to the trajectory, where $K_{m}$ are modified Bessel functions and $m=0(m=1)$ must be selected for directions parallel (perpendicular) to the velocity vector $\mathbf{v}$. For low photon energies $\hbar \omega \lesssim 1 \mathrm{eV}$ and 
small distances $(R \sim$ few $\mathrm{nm})$, the perpendicular component becomes dominant and the Bessel function in this expression can be approximated as $K_{1}(\theta) \approx 1 / \theta$, even for electron velocities as small as $v \sim c / 100$ (i.e., $\sim 25 \mathrm{eV}$ electrons), leading to a frequency-independent field amplitude $2 e /(v R)$.

The transient evanescent field of the electron presents a fluence $\mathcal{F}=(c / 2 \pi) \int d t\left|\mathbf{E}^{\operatorname{ext}}(\mathbf{r}, t)\right|^{2}=$ $\left(c / 4 \pi^{2}\right) \int d \omega\left|\mathbf{E}^{\operatorname{ext}}(\mathbf{r}, \omega)\right|^{2}$. In order to estimate the effect of this field on sample excitations, we consider an optical mode of frequency $\omega_{0}$ and lifetime $\tau$, which allows us to define an effective fluence $\mathcal{F}^{\mathrm{eff}}=\left(c / 4 \pi^{2}\right) \int d \omega\left|\mathbf{E}^{\mathrm{ext}}(\mathbf{r}, \omega)\right|^{2} /\left[1+4\left(\omega-\omega_{0}\right)^{2} \tau^{2}\right]$ obtained by weighting the spectral field components with the corresponding Lorentzian line shape of the resonance. As we argue above, $\mathbf{E}^{\mathrm{ext}}(\mathbf{r}, \omega)$ is nearly independent of $\omega$ for the low resonance energies and small distances under consideration $\left(\omega_{0} R \ll v\right)$, thus allowing us to obtain $\mathcal{F}^{\text {eff }} \approx e^{2} c /\left(2 \pi v^{2} R^{2} \tau\right)$ (Figure 1b, blue curve). Now, it is convenient to compare this estimate with a typical ultrashort Gaussian light pulse ( $2 \operatorname{Re}\left\{E \mathrm{e}^{-\mathrm{i} \omega_{0} t-t^{2} / \Delta^{2}}\right\}$ field profile) of $\Delta=100 \mathrm{fs}$ duration and the same fluence $c E^{2} \Delta / 8 \pi=\mathcal{F}^{\text {eff }}$; this leads to an equivalent light field peak amplitude $E \sim 10^{7} \mathrm{~V} / \mathrm{m}$ at a distance $R=1 \mathrm{~nm}$ for $25 \mathrm{eV}$ electrons acting on an excitation of width $\hbar \tau^{-1}=10 \mathrm{meV}$ (Figure 1b, blue line and right vertical axis), which is sufficient to produce substantial nonlinear effects in several materials, and in particular, gold and graphene, as we discuss below.

Highly doped graphene inherits a strong nonlinear response from its conical electronic band structure with constant Fermi velocity $v_{\mathrm{F}} \approx c / 300{ }^{23-25}$ Additionally, this material exhibits electrically tunable infrared plasmons ${ }^{26-33}$ that have been argued to boost the nonlinear response to unpredecedented levels. ${ }^{34}$ Following a previously reported analytical description of plasmon-mediated nonlinear response of graphene islands, ${ }^{35}$ in excellent agreement with quantum-mechanical calculations at the same level of theory used in the present work, we can readily quantify the external optical field amplitude $E$ that needs to be applied to a nanographene structure in order to produce a nonlinear dipole strength similar to the linear one; here, we present a succinct description of the procedure described in the Methods sec- 
tion: from the condition $\left|\alpha_{\omega}^{11} E\right|=\left|\alpha_{\omega}^{31} E^{3}\right|$, where $\alpha_{\omega}^{11}$ and $\alpha_{\omega}^{31}$ are the linear and third-order polarizabilities at frequency $\omega$, we find $|E|^{2} \approx 1.39 E_{\mathrm{F}}{ }^{3 / 2} D^{1 / 2} /\left(e^{3} v_{\mathrm{F}}^{2} \tau^{3}\right)$ at the peak energy $0.60 e \sqrt{E_{\mathrm{F}} / D}$ of the dipolar plasmon sustained by a graphene disk supported on silicon $(\epsilon=12.1)$ with side length $D$ and Fermi energy $E_{\mathrm{F}}$. This rough estimate (Figure 1b, grey line, for $E_{\mathrm{F}}=1 \mathrm{eV}$ ) predicts that the onset of nonlinear response in graphene nanoislands is below the effective fluence provided by a $\sim 25 \mathrm{eV}$ electron at distances $\lesssim 5 \mathrm{~nm}$ from the beam. We note that the actual onset value depends on $E_{\mathrm{F}}, \tau$, sample morphology, and orientation of the electron trajectory, which could be further adjusted to make it even lower.

From the measured third-order nonlinear response of gold, ${ }^{36}$ we estimate $E^{2}=\left|\alpha_{\omega}^{11} / \alpha_{\omega}^{31}\right| \sim$ $7 \times 10^{15} \mathrm{~V}^{2} / \mathrm{m}^{2}$ for metal spheres at $532 \mathrm{~nm}$ light wavelength. This field onset, which is shown in Figure 1b for comparison, lies above the equivalent optical field supplied by a $25 \mathrm{eV}$ electron. However, we expect significantly lower onset fields due to near-field enhancement in gold tips and narrow gaps, where nonlinear effects could perhaps be effectively triggered by free electrons within the energy range considered here.

Nonlinear CL Emission from Graphene Nanohexagons. We apply the quantummechanical atomistic formalism outlined in Methods to study the response of a hexagonal graphene nanoisland with exclusively armchair edge terminations lying in the $x$ - $y$ plane, consisting of $N=546$ carbon atoms $(\approx 4.5 \mathrm{~nm}$ diameter $)$, and doped to a Fermi energy $E_{\mathrm{F}}=1 \mathrm{eV}$. To examine the nonlinear response, we consider the interaction with a point charge $q$ (e.g., $q=-e$ for an electron) that moves parallel to and $z_{0}=0.5 \mathrm{~nm}$ above the graphene plane with velocity $c / 100(\approx 25 \mathrm{eV}$ energy for electrons), as illustrated in Figure 2a. The probe takes only $\sim 1.5 \mathrm{fs}$ to fly over the island, but its effects, quantified by the induced dipole $p(t)$ (oriented along the beam direction due to the symmetry of the system), are observed to last much longer (roughly over a time scale dictated by the relaxation time $\tau$, in this case $\approx 66 \mathrm{fs}$ ), as shown in Figure $2 \mathrm{c}$, where we normalize the dipole to the charge $q$ in order to directly visualize any departure from the linear regime. We find that the normalized dipole does not present observable variations within the scale of the plot when comparing 

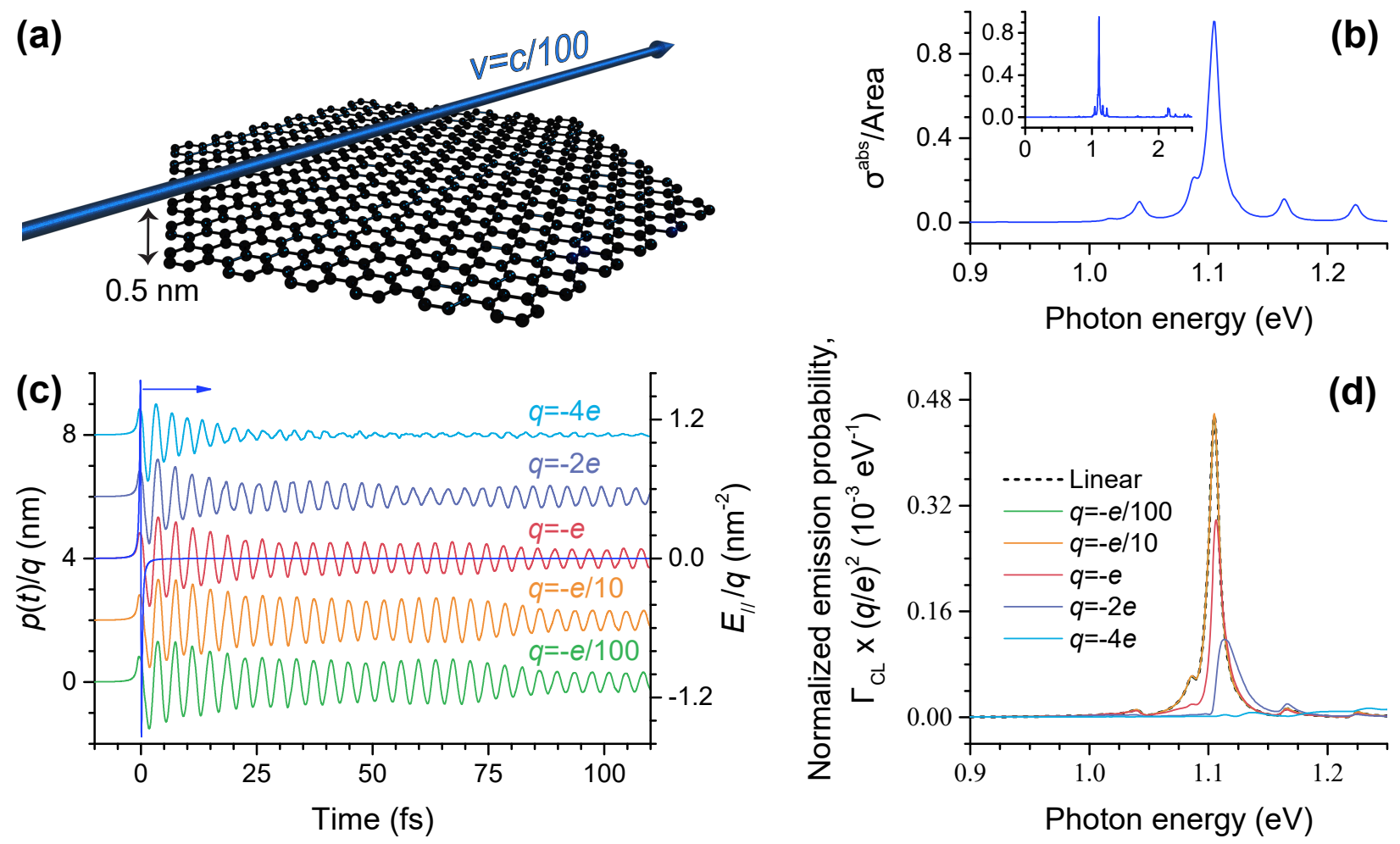

Figure 2: Nonlinear plasmonic CL emission from a graphene nanoisland. (a) Schematic illustration of an armchair-edged hexagonal graphene nanoisland $(546 \mathrm{C}$ atoms, $4.55 \mathrm{~nm}$ diameter) interacting with a point charge $q$ passing parallel to and $0.5 \mathrm{~nm}$ above the graphene plane. (b) Absorption spectrum (cross section $\sigma^{\text {abs }} \approx(4 \pi \omega / c) \operatorname{Im}\left\{\alpha_{\omega}^{11}\right\}$ normalized to hexagon area) for normally incident light. (c) Time dependence of the dipole moment $p(t)$ induced on the island by probes of different charge $q$ moving with velocity $c / 100(\approx 25 \mathrm{eV}$ energy for electrons). For comparison, we show the external electric field component $E_{\|}$ parallel to the beam direction produced by the electron at the hexagon center (right scale). The electron passes above this point at $t=0$. (d) CL emission probability normalized to the squared probe charge $q^{2}$. We set the Fermi energy to $E_{\mathrm{F}}=1 \mathrm{eV}$ (doping charge $Q \sim 16 e$ ) and the damping to $\hbar \tau^{-1}=10 \mathrm{meV}$. 
results for $q=-e / 10$ and $q=-e / 100$, indicating that these small charges are still unable to trigger a nonlinear response. However, we find $p(t)$ to decay substantially faster over time for $q=-e$ (i.e., for an electron). This effect, which appears to evolve nonmonotonically with increasing $q$, effectively saturates the response and becomes rather strong at $q=-4 e$. In all cases, $p(t)$ oscillates with a period of $\sim 3.8 \mathrm{fs}$, in good agreement with the optical period of the $1.1 \mathrm{eV}$ dominant plasmon revealed in the linear optical absorption spectrum of the structure (Figure 2b), which is confirmed in the CL emission spectra (Figure 2d): the normalized intensities for $q=-e / 10$ and $q=-e / 100$ cannot be distinguished from the result predicted by linear response theory on the scale of the plot and follow a similar spectral profile as the absorption; however, the results for $q=-e$ already reveal significant spectral variations (attenuation, overall blue shift, and spectral reshaping), which become dramatic as $q$ increases further.

We note that, as explained in Methods, our simulations are performed in the nonrecoil approximation, so that the probe is unperturbed by its interaction with the graphene nanoislands; however, recoil corrections for an electron probe are anticipated to introduce only small modifications, such as frequency shifts of the emission features. ${ }^{17}$ Additionally, these results are general for a point charge with constant velocity, and thus apply also to heavier particles such as ions, for which the nonrecoil approximation should be quantitatively very accurate down to low speeds, even below that considered here. Incidentally, given the small size of the graphene island compared to the wavelength, the angular profile of emission should be dipolar. Interestingly, the saturation and spectral shifts in CL emission resemble those arising due to the optical Kerr nonlinearity for plasmons that are resonantly driven by intense ultrashort light pulses; such effects become more important for pulses of longer duration, eventually approaching a bistable regime for $\mathrm{cw}$ illumination. ${ }^{34}$ Our findings confirm that a moving point probe can trigger nonlinearities akin to those produced by intense ultrashort optical pulses, with the probe velocity defining the strength of the nonlinear interaction. Size, Doping, and Beam-Energy Dependence. Examining graphene islands of different 

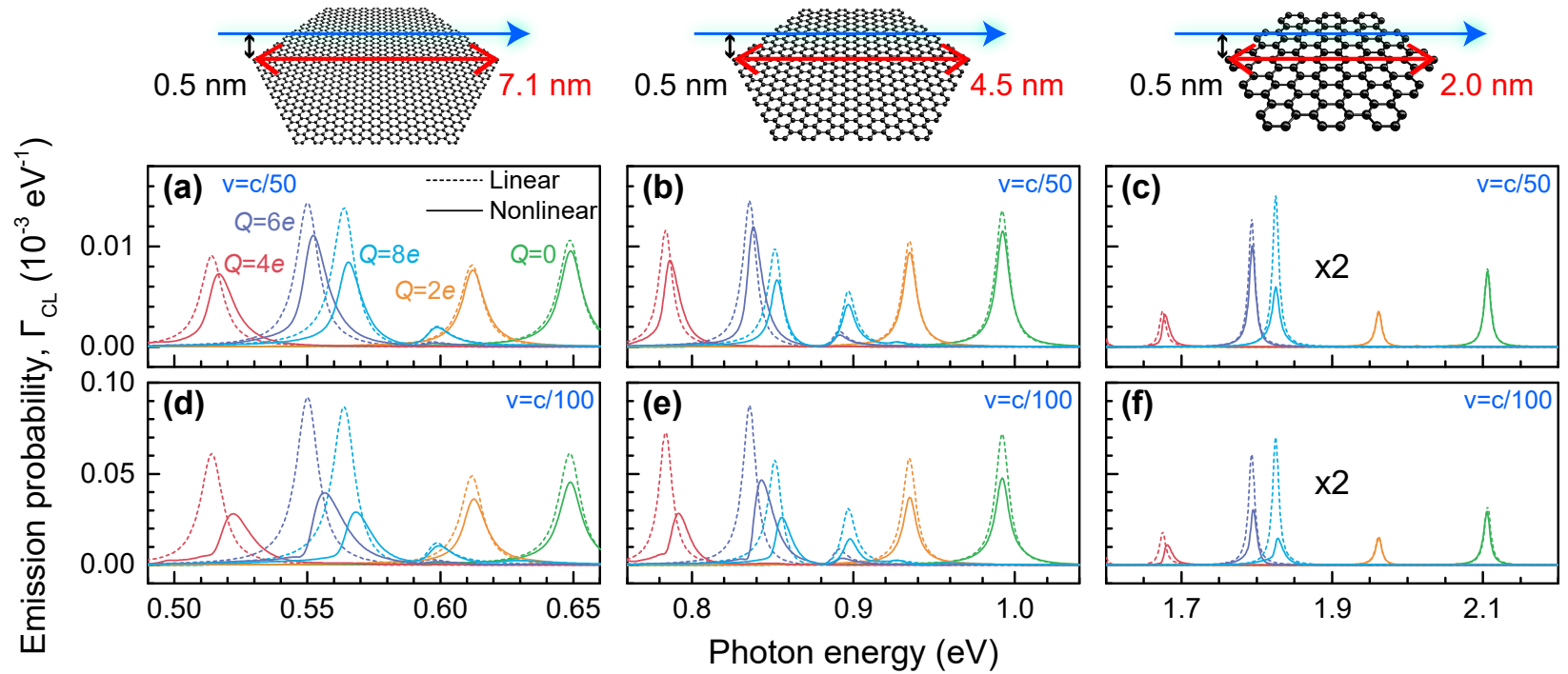

Figure 3: Size and doping dependence of free-electron-induced nonlinear CL. Simulated CL spectra for graphene nanohexagons with C-to-C diameters of (a,d) $7.11 \mathrm{~nm}(1302$ $\mathrm{C}$ atoms), (b,e) $4.55 \mathrm{~nm}$ (546 atoms), and (c,f) $1.99 \mathrm{~nm}$ (114 atoms). Electrons are impinging parallel to and $0.5 \mathrm{~nm}$ above the graphene plane with an energy of either (a-c) $100 \mathrm{eV}$ $(v \approx c / 50)$ or $(\mathrm{d}-\mathrm{f}) 25 \mathrm{eV}(v \approx c / 100)$. The structures are doped with an additional charge $Q$ (see labels in (a)). Nonlinear time-domain simulations (solid curves) are compared to results obtained from linear response theory (dashed curves).

diameter $D$ (see upper insets in Figure 3), we corroborate the expected scaling of the intensity associated with the onset of nonlinear effects as $\propto E_{\mathrm{F}}{ }^{3 / 2} D^{1 / 2}$. Indeed, for a fixed doping charge $Q$, we have ${ }^{37} E_{\mathrm{F}} \propto Q^{1 / 2} / D$, therefore resulting in a $Q^{1 / 2} / D$ overall dependence of the onset, so that stronger nonlinear effects are produced in larger hexagons for an equal number of doping charges ( $c f$. dashed (linear) and solid (nonlinear) curves of the same color in the three structures considered in Figure 3) or when increasing $Q$ in each of the hexagons. The scaling of the probe field amplitude as $\propto 1 / v$ also affects the magnitude of the generated nonlinear effects, which are substantially increased when moving from $v=c / 50$ (upper plots in Figure 3) to $v=c / 100$ (lower plots). Similar to the effects observed for the hexagon examined in Figure 2, the nonlinear response produces plasmon blue shifts and attenuation of the CL emission intensity for all of the hexagon sizes, electron energies, and doping conditions considered in Figure 3. Generally, we observe more pronounced shifts in the CL emission peaks for the largest graphene island, while those of the smallest island tend to saturate. 
Indeed, while plasmons are redshifted with increasing size as a result of the increase in aspect ratio of the planar graphene island, the detailed spectral dependence on $D$ and $Q$ is strongly influenced by quantum-mechanical effects for the small islands under consideration. ${ }^{22}$ In particular, the emergence of gaps in electronic structure for these structures endow them with a high sensitivity to minute changes in electron population, particularly for the smallest island resembling a large polycyclic aromatic hydrocarbon; besides adding or removing an individual electron, substantial changes in the plasmon resonance energies and associated nonlinearities in the CL spectra can manifest from the re-distribution of electron population for a raised graphene electron temperature, as we explore in Figure S1 in the Supplementary Information (SI).

Practical Schemes for Nonlinear CL Measurements. The analysis of the CL intensities performed above as a function of probe charge $q$ is not practical in actual experiments involving low-energy electrons. Instead, we propose to study the way in which the CL emission spectra evolve when varying more easily controllable e-beam parameters. The latter can affect the strength of the electron field actually acting on the sample, effectively allowing us to explore the transition between linear and nonlinear regimes. Specifically, we consider the spectral dependence either on the lateral position of the e-beam for fixed electron energy (Figure 4a) or on electron energy for fixed e-beam position (Figure 4b).

The dependence on lateral impact parameter $d$ shows a nonmonotonic evolution of both the nonlinear plasmon blue shift and its associated emission intensity, which do however follow a similar pattern, reaching a maximum of $\sim 10 \mathrm{meV}$ at intermediate positions of the e-beam. This result confirms the intuition that the contribution of the dominant transversal field of the electron suffers sign cancellations when trying to excite the plasmon polarized along the e-beam direction with a central symmetric trajectory. Also, an outer trajectory produces comparatively weaker polarization and blue shift (e.g., $\sim 3 \mathrm{meV}$ for $d=4 \mathrm{~nm}$ ) because it does not overlap the bulk of the island. Consequently, an optimal interaction is found in Figure 4a in an intermediate configuration, for which nonlinear effects are maximized. 

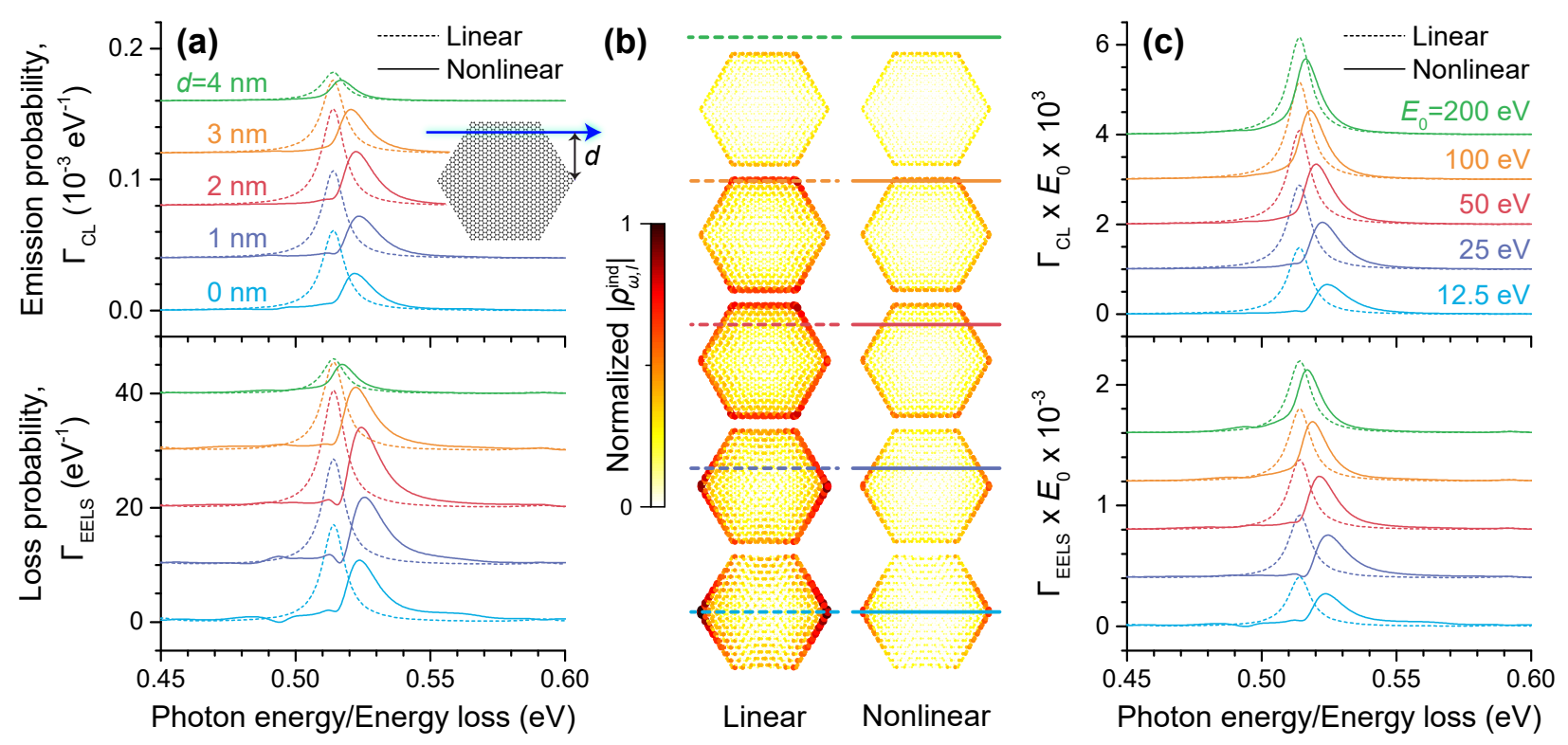

Figure 4: Resolving electron-induced nonlinearities by laterally displacing the e-beam or changing its energy. (a) Evolution of the CL emission (upper panel) and electron energy-loss (lower panel) spectra from a hexagon consisting of $1302 \mathrm{C}$ atoms and doping charge $Q=4 e$ for an e-beam with fixed energy $E_{0}=25 \mathrm{eV}$ running parallel to and $0.5 \mathrm{~nm}$ above the graphene plane when varying the lateral impact parameter $d$ (see inset and labels). Solid (dashed) curves correspond to nonlinear (linear) simulations. (b) Spatial maps of the associated induced charge $\left|\rho_{\omega, l}^{\text {ind }}\right|$ at the carbon sites $\mathbf{R}_{l}$ for the electron trajectories considered in (a), indicated by color-coded horizontal dashed (solid) lines, as obtained from linear (nonlinear) simulations. In each plot, the frequency $\omega$ is taken at the maximum of the corresponding spectrum in (a); the symbol size and color strength indicates the magnitude of $\left|\rho_{\omega, l}^{\text {ind }}\right|$ normalized to the overall maximum in the ten plots. (c) CL and EELS spectra obtained by fixing $d=2 \mathrm{~nm}$, varying the electron energy $E_{0}$, and scaling the CL emission and EELS probabilitfies ( $\Gamma_{\mathrm{CL}}$ and $\left.\Gamma_{\mathrm{EELS}}\right)$ by $10^{3} E_{0}$ and $10^{-3} E_{0}$, respectively. 
Additionally, we find that the nonlinearity exhibits a monotonic increase with decreasing electron energy, in agreement with the low-velocity $1 / v^{2}$ scaling of the electron field intensity. Actually, linear simulations produce roughly energy-independent spectral line shapes that have similar intensity when multiplied by the electron energy $E_{0}$ (Figure $\left.4 \mathrm{~b}\right)$. In contrast, nonlinear simulations reveal an increasing departure from this regime as the electron energy is reduced, with blue shifts in the $\sim 3-10 \mathrm{meV}$ range for the energies considered.

Nonlinear Response Revealed through EELS. The CL emission only accounts for a fraction of the energy lost by the electron probe. The ratio between CL and EELS probabilities is in fact similar to the ratio between the cross sections for optical elastic scattering and extinction (see Figure S2 in SI), which is $\sim 10^{-5}$ under the conditions of Figure 2. For simplicity, we estimate the EELS probability from the Fourier transform of the induced field, using the same expression as in the weak coupling regime, under the assumption that multi-

ple nonlinear interactions do not produce significant re-distribution of energy-loss events in the electron spectra (see Methods). We find that the calculated EELS spectra in Figure 4 (lower panels) show similar spectral profiles as the CL emission (upper panels), except for the much larger probability and some small relative variations between linear and nonlinear theories depending on electron energy and impact parameter. These conclusions are general and apply to the rest of the structures considered in this work (see, for example, Figure S3 in the SI, which shows EELS spectra under the same conditions as in Figure 3).

\section{Concluding Remarks}

In summary, we have shown that the evanescent electromagnetic field carried by low-energy electrons acts on the plasmon modes supported by graphene nanostructures in a similar way as intense ultrashort laser pulses, capable of driving strong nonlinear response that should be observable through saturation and frequency shifts of the resulting CL emission and EELS peaks. The electron energy range under consideration $(<200 \mathrm{eV})$ is commonly used in low- 
energy electron microscopes (LEEMs), which can currently reach a spatial resolution down to $<10 \mathrm{~nm} .{ }^{38,39}$ These types of setups could be used to investigate the nonlinear response of individual graphene nanoislands, such as chemically synthesized quantum dots. ${ }^{40}$ Alternatively, the nonlinear response of graphene nanoislands could be examined by depositing them on a dielectric surface and analyzing either the EELS probability or the CL emission associated with low-energy electrons that are grazingly scattered from such surface. Short electron-graphene distances such as those considered in this work $\sim 0.5 \mathrm{~nm}$ are expected under grazing-incidence surface electron scattering in the context of LEEM ${ }^{38,39}$ and elastic low-energy electron diffraction ${ }^{41}$ (LEED), which is routinely employed to resolve crystal surface structures. An important aspect of the interaction between the graphene nanoisland and the electron beam relates to the lateral extension of the latter, as we expect that stronger nonlinear effects arise when the focal spot is comparable to or smaller than the size of the island; this condition can be achieved using either LEEMs ${ }^{38,39}$ or secondary-electron microscopes (SEMs) equipped with CL acquisition systems ${ }^{42}$ to explore islands in the fewnm range. ${ }^{40}$ The rate of photon emission and loss events is also an important parameter to be taken into account for the experimental observation of the effects here predicted. In particular, from the CL and EELS spectra presented in Figure 4, we find $\sim 10^{-7}$ photons and $\sim 10^{-2}$ loss events associated with the main plasmon feature per incident electron; this CL yield is certainly challenging (although higher rates could be achieved under grazing incidence, which allows the electron to constructively interact with a large number of islands deposited on a substrate), while the EELS probability is sufficiently large to be comfortably measured using currently available setups. Obviously, an increase in the nonlinear response should arise when using highly charged ions as probes instead of electrons, for example under glancing incidence on a surface where the islands are deposited, following similar methods as in previous studies of ion-surface interaction. ${ }^{43}$ CL emission and EELS spectra convey similar information on the nonlinear response, which could be complemented by simultaneous interaction with strong light pulses, as recently proposed in the context of PINEM. ${ }^{44}$ 
The possibility of inducing strong nonlinear effects on the nanoscale using a single lowenergy electron offers an appealing alternative to conventional nonlinear optical experiments relying on ultrafast and high power lasers. Bridging current research activities in electron spectroscopy and nonlinear optics, this concept adds a new dimension to CL and EELS, while elucidating nonlinear dynamics of nanostructured materials without risking optically-induced damage by high-fluence pulses and enabling a gain in spatial resolution by several orders of magnitude when comparing electron- and light-beam focal spots. Single-free-electroninduced nonlinearity further suggests a mechanism to blockade the excitation of nanoscale optical resonances, potentially enabling an electron-based analogue of ultrafast all-optical switching, and warranting future studies on the quantum information that can be encoded and transferred between nanoscale optical resonators and individual free electrons.

\section{Methods}

Analytical Comparison of Electron- and Light-Induced Nonlinearities. The nonlinear optical response of light-driven plasmon resonances supported by graphene nanostructures of $\gtrsim 10 \mathrm{~nm}$ lateral size can be described in a semi-analytical fashion following previously described methods, ${ }^{35}$ which we apply here to obtain an estimate for the equivalent optical fluence associated with the passage of a free electron, as shown in Figure 1b. We consider for simplicity a graphene disk of diameter $D$ and Fermi energy $E_{\mathrm{F}}$ deposited on silicon $(\epsilon=12.1)$ and described by the local Drude conductivity $\sigma(\omega)=\left(\mathrm{i} e^{2} E_{\mathrm{F}} / \pi \hbar^{2}\right) /\left(\omega+\mathrm{i} \tau^{-1}\right)$ with inelastic lifetime $\tau$. We focus on the disk dipolar plasmon, whose energy is $\hbar \omega \approx e \sqrt{E_{\mathrm{F}} /\left(-\pi \eta_{1} \epsilon D\right)} \approx$ $0.60 e \sqrt{E_{\mathrm{F}} / D}$ with $\eta_{1}=-0.073 .{ }^{35}$ Now, we estimate the optical electric field amplitude $E$ that is required to trigger large nonlinear effects from the condition that the induced dipole components $\left|\alpha_{\omega}^{11} E\right|$ and $\left|\alpha_{\omega}^{31} E^{3}\right|$ associated with linear and third-order Kerr nonlinear response, respectively, have the same magnitude. Using analytical expressions for the respective polarizabilities $\alpha_{\omega}^{11}$ (eq 29a in Ref. 35) and $\alpha_{\omega}^{31}$ (eq 29c in Ref. 35) under the assumption 
$\omega \tau \gg 1$, we obtain the condition $|E|^{2}=\left|\alpha_{\omega}^{11} / \alpha_{\omega}^{31}\right|=C \times \hbar E_{\mathrm{F}}{ }^{3 / 2} D^{1 / 2} /\left(e^{3} v_{\mathrm{F}}^{2} \tau^{3}\right)$, where the coefficient $C=8 \sqrt{-\pi \eta_{1} \epsilon} \zeta^{2} / 9 \tilde{\xi} \approx 1.39$ is obtained from the values $\zeta=0.85$ and $\tilde{\xi}=0.77$ tabulated in Table I of Ref. 35 .

Quantum-Mechanical Description of e-Beam Interactions with Nanographenes.

We consider a graphene nanoisland lying in the $z=0$ plane and interacting with a point charge $q$ (e.g., $q=-e$ for an electron) that moves with velocity $\mathbf{v}$ along the trajectory $\mathbf{r}=\mathbf{r}_{\mathrm{e}}+\mathbf{v} t$ and passes by the position $\mathbf{r}_{\mathrm{e}}$ at time $t=0$. We take $v \ll c$, allowing us to safely neglect retardation effects. Additionally, we adopt the nonrecoil approximation by maintaining $\mathbf{v}$ constant, thus disregarding changes in $\mathbf{v}$ arising from energy exchanges with the sample; this is a reasonable assumption in the present study, where the probe kinetic energy is large compared with the emitted photon energy $\hbar \omega$. Although we consider doped graphene islands, we neglect the effect of additional charge carriers on the trajectory of the probe, which is a reasonable approximation for charge transfer from a substrate (i.e., when extra graphene carriers are electrically neutralized by opposite charges on the substrate). The external potential can thus be written as

$$
\phi^{\mathrm{ext}}(\mathbf{r}, t)=\frac{q}{\left|\mathbf{r}-\mathbf{r}_{\mathrm{e}}-\mathbf{v} t\right|}
$$

Following previous work, ${ }^{34}$ we characterize the response of the graphene nanoisland to the probe through the induced charge density $\rho_{l}^{\text {ind }}(t)$ associated with the out-of-plane $2 \mathrm{p}$ orbitals $|l\rangle$ located at the carbon sites $\mathbf{R}_{l}=\left(x_{l}, y_{l}\right)$ (one orbital per carbon atom). The induced charge is computed from the single-particle density matrix $\rho$ according to $\rho_{l}^{\text {ind }}=-2 e \sum_{l}\left(\rho_{l l}-\rho_{l l}^{0}\right)$, with a factor of 2 accounting for spin degeneracy and the time dependent density matrix elements $\rho_{l l^{\prime}}$ in the site basis governed by ${ }^{34}$

$$
\mathrm{i} \hbar \frac{\partial \rho_{l l^{\prime}}}{\partial t}=\sum_{l^{\prime \prime}}\left(H_{l l^{\prime \prime}}^{\mathrm{TB}} \rho_{l^{\prime \prime} l^{\prime}}-\rho_{l l^{\prime \prime}} H_{l^{\prime \prime} l^{\prime}}^{\mathrm{TB}}\right)-e\left(\phi_{l}-\phi_{l^{\prime}}\right) \rho_{l l^{\prime}}-\frac{\mathrm{i} \hbar}{\tau}\left(\rho_{l l^{\prime}}-\rho_{l l^{\prime}}^{0}\right) .
$$

In this equation of motion, the kinetic tight-binding Hamiltonian $H^{\mathrm{TB}}$ consists of a $-2.8 \mathrm{eV}$ 
nearest-neighbor hopping ${ }^{37}$ and yields energy eigenvalues $\hbar \varepsilon_{j}$ satisfying $\hbar \varepsilon_{j}|j\rangle=H^{\mathrm{TB}}|j\rangle$, such that the eigenstates $|j\rangle$ are mapped onto the site basis by real-valued expansion coefficients $a_{j l}$ according to $|j\rangle=\sum_{l} a_{j l}|l\rangle$. The self-consistent electrostatic potential $\phi_{l}=$ $\phi_{l}^{\text {ext }}+\sum_{l^{\prime}} v_{l l^{\prime}} \rho_{l^{\prime}}^{\text {ind }}$ describes the external potential (eq 1 ) and electron-electron Hartree interaction in graphene, with $v_{l l^{\prime}}$ denoting the spatial dependence of the Coulomb repulsion between electrons at carbon sites $\mathbf{R}_{l}$ and $\mathbf{R}_{l^{\prime}}$. ${ }^{45}$ Additionally, the inelastic scattering of graphene electrons is treated by relaxing the system with a phenomenological time $\tau$ $\left(\hbar \tau^{-1}=10 \mathrm{meV}\right.$ throughout this work) to the equilibrium density matrix $\rho_{l l^{\prime}}^{0}=\sum_{j} f_{j} a_{j l} a_{j l^{\prime}}$, constructed from the state occupation factors $f_{j}$. When the graphene doping is specified by a Fermi energy $E_{\mathrm{F}}$, the state occupation factors $f_{j}$ follow the Fermi-Dirac statistics, such that $f_{j}=\left[1+\mathrm{e}^{\left(\varepsilon_{j}-E_{\mathrm{F}}\right) / k_{\mathrm{B}} T}\right]^{-1}$, choosing an electron temperature $T=0$ throughout this work except in Figure S1 of the SI. When a doping charge $Q$ is specified instead of $E_{\mathrm{F}}$, we determine $f_{j}$ by sequentially filling the lowest-energy unoccupied states with up to two spin-degenerate electrons (imposing equal values for degenerate states) until reaching a total of $2 \sum_{j} f_{j}=N-Q / e$ electrons, where $N$ is the number of carbon atoms.

Time-Domain Simulations. Starting at a time for which the probe charge is still far away from the graphene structure, direct numerical integration of eq 2 yields the density matrix at an arbitrary time $t$, allowing us to extract the time-evolving induced dipole moment $\mathbf{p}(t)=\sum_{l} \rho_{l}^{\text {ind }} \mathbf{R}_{l}$ from which CL spectra are obtained through Fourier analysis. Specifically, we decompose $\mathbf{p}(t)=\int(d \omega / 2 \pi) \mathrm{e}^{-\mathrm{i} \omega t} \mathbf{p}_{\omega}$ and calculate the frequency-resolved emission probability (photons emitted per unit time and frequency range) from the far-field integral of the Poynting vector as ${ }^{6}$

$$
\Gamma_{\mathrm{CL}}=\frac{2 \omega^{3}}{3 \pi \hbar c^{3}}\left|\mathbf{p}_{\omega}\right|^{2}
$$

For EELS, we follow methods previously developed in the weak-coupling regime, ${ }^{6}$ which should provide a reasonable estimate of the transmitted spectra, although more elaborate 
analysis beyond the scope of this work is needed to conclusively elucidate the role of multiple nonlinear interactions in redistributing energy losses in the resulting spectra. In the nonretarded limit, the weak-coupling EELS probability reduces to

$$
\Gamma_{\mathrm{EELS}}=\frac{e}{\pi \hbar} \int d t \operatorname{Re}\left\{\mathrm{e}^{-\mathrm{i} \omega t} \partial_{z} \phi^{\mathrm{ind}}\left(\mathbf{r}_{\mathrm{e}}+\mathbf{v} t, \omega\right)\right\}
$$

where the induced potential

$$
\phi^{\text {ind }}(\mathbf{r}, \omega)=\sum_{l} \frac{\rho_{\omega, l}^{\text {ind }}}{\left|\mathbf{r}-\mathbf{R}_{l}\right|}
$$

is computed from the frequency-space induced charge $\rho_{\omega, l}^{\text {ind }}=\int d t \rho_{l}^{\text {ind }}(t) \mathrm{e}^{\mathrm{i} \omega t}$. Direct insertion of eq 5 into eq 4 yields the more explicit expressions

$$
\begin{aligned}
& \Gamma_{\mathrm{EELS}}^{\perp}(\omega)=\frac{2 e}{\pi \hbar v} \sum_{l} K_{0}\left(\frac{\omega}{v}\left|\mathbf{R}_{l}-\mathbf{R}_{\mathrm{e}}\right|\right) \operatorname{Im}\left\{\rho_{\omega, l}^{\text {ind }}\right\} \\
& \Gamma_{\mathrm{EELS}}^{\|}(\omega)=\frac{2 e}{\pi \hbar v} \sum_{l} K_{0}\left(\frac{\omega}{v} \sqrt{\left(y_{l}-d\right)^{2}+z_{\mathrm{e}}^{2}}\right) \operatorname{Im}\left\{\rho_{\omega, l}^{\text {ind }} \mathrm{e}^{-\mathrm{i} \omega x_{l} / v}\right\}
\end{aligned}
$$

for perpendicular $\left(\mathbf{v} \| \hat{\mathbf{z}}, \mathbf{r}_{\mathrm{e}}=\mathbf{R}_{\mathrm{e}}=\left(x_{\mathrm{e}}, y_{\mathrm{e}}\right)\right)$ and parallel $\left(\mathbf{v} \| \hat{\mathbf{x}}, \mathbf{r}_{\mathrm{e}}=\left(0, d, z_{\mathrm{e}}\right)\right)$ trajectories, where $K_{0}$ is a modified Bessel function.

Linear Response. Retaining linear terms in the perturbation expansion of eq 2 and, in the spirit of the random-phase approximation (RPA), replacing the external potential by the self-consistent potential, one obtains the expression ${ }^{45,46}$

$$
\rho_{\omega, l}^{\text {ind }}=\sum_{l^{\prime}} \chi_{l l^{\prime}} \phi_{\omega, l^{\prime}}^{\mathrm{ext}}
$$

for the induced charge, where $\chi$ is the self-consistent susceptibility expressed in terms of the noninteracting susceptiblity

$$
\chi_{l l^{\prime}}^{0}=\frac{2 e^{2}}{\hbar} \sum_{j j^{\prime}}\left(f_{j^{\prime}}-f_{j}\right) \frac{a_{j l} a_{j^{\prime} l} a_{j l^{\prime}} a_{j^{\prime} l^{\prime}}}{\omega+\mathrm{i} / 2 \tau-\left(\varepsilon_{j}-\varepsilon_{j^{\prime}}\right)}
$$


according to $\chi=\chi_{0} \cdot\left(1-\chi_{0} \cdot v\right)^{-1}$ for matrices indexed by $l$, and $v_{l l^{\prime}}$ is the abovementioned Coulomb interaction. The Fourier transform of the external potential $\phi_{\omega, l}^{\text {ext }}=$ $\int d t \phi^{\operatorname{ext}}\left(\mathbf{R}_{l}, t\right) \mathrm{e}^{\mathrm{i} \omega t}$ (see eq 1) admits the analytical expressions $\phi_{\omega, l}^{\text {ext }}=(2 q / v) K_{0}\left[(\omega / v)\left|\mathbf{R}_{l}-\mathbf{R}_{\mathrm{e}}\right|\right]$ and $\phi_{\omega, l}^{\text {ext }}=(2 q / v) K_{0}\left[(\omega / v) \sqrt{\left(y_{l}-d\right)^{2}+z_{\mathrm{e}}^{2}}\right] \mathrm{e}^{\mathrm{i} \omega x_{l} / v}$ for perpendicular and parallel trajectories, respectively, which we use to obtain $\rho_{\omega, l}^{\text {ind }}$ from eq 7 , and this in turn to calculate CL and EELS probabilities through eqs 3 and 6.

\section{Supplementary Information}

The Supporting Information is available free of charge at https://pubs.acs.org/doi/10.1021/acs.nanolett.xxx

We show the dependence of the CL probability on the graphene electron temperature, the relative magnitude of CL and EELS probabilities, and the EELS counterpart of Figure 3.

\section{Acknowledgments}

This work has been supported in part by the European Research Council (Advanced Grant 789104-eNANO), the Spanish MINECO (MAT2017-88492-R and SEV2015- 0522), the European Commission (Graphene Flagship 696656), the Catalan CERCA Program, and Fundació

Privada Cellex. The Center for Nano Optics is financially supported by the University of Southern Denmark (SDU 2020 funding). J.D.C was supported by VILLUM Fonden (grant No. 16498).

\section{References}

(1) Born, M.; Wolf, E. Principles of Optics: Electromagnetic Theory of Propagation, Interference and Diffraction of Light; Cambridge University Press: Cambridge, 1999. 
(2) Huth, F.; Govyadinov, A.; Amarie, S.; Nuansing, W.; Keilmann, F.; Hillenbrand, R. Nano-FTIR Absorption Spectroscopy of Molecular Fingerprints at $20 \mathrm{~nm}$ Spatial Resolution. Nano Lett. 2012, 12, 3973-3978.

(3) Krivanek, O. L.; Lovejoy, T. C.; Dellby, N.; Aoki, T.; Carpenter, R. W.; Rez, P.; Soignard, E.; Zhu, J.; Batson, P. E.; Lagos, M. J.; Egerton, R. F.; Crozier, P. A. Vibrational Spectroscopy in the Electron Microscope. Nature 2014, 514, 209-214.

(4) Lagos, M. J.; Trügler, A.; Hohenester, U.; Batson, P. E. Mapping Vibrational Surface and Bulk Modes in a Single Nanocube. Nature 2017, 543, 529-532.

(5) Senga, R.; Suenaga, K.; Barone, P.; Morishita, S.; Mauri, F.; Pichler, T. Position and Momentum Mapping of Vibrations in Graphene Nanostructures in the Electron Microscope. Nature 2019, 247-250.

(6) García de Abajo, F. J. Optical Excitations in Electron Microscopy. Rev. Mod. Phys. 2010, 82, 209-275.

(7) Nelayah, J.; Kociak, M.; O. Stéphan,; García de Abajo, F. J.; Tencé, M.; Henrard, L.; Taverna, D.; Pastoriza-Santos, I.; Liz-Marzán, L. M.; Colliex, C. Mapping Surface Plasmons on a Single Mmetallic Nanoparticle. Nat. Phys. 2007, 3, 348-353.

(8) Vesseur, E. J. R.; de Waele, R.; Kuttge, M.; Polman, A. Direct Observation of Plasmonic Modes in $\mathrm{Au}$ Nanowires Using High-Resolution Cathodoluminescence Spectroscopy. Nano Lett. 2007, 7, 2843-2846.

(9) Bosman, M.; Keast, V. J.; Watanabe, M.; Maaroof, A. I.; Cortie, M. B. Mapping Surface Plasmons at the Nanometre Scale with an Electron Beam. Nanotechnology 2007, 18, 165505.

(10) Rossouw, D.; Botton, G. A. Plasmonic Response of Bent Silver Nanowires for Nanophotonic Subwavelength Waveguiding. Phys. Rev. Lett. 2013, 110, 066801. 
(11) Guzzinati, G.; Beche, A.; Lourenco-Martins, H.; Martin, J.; Kociak, M.; Verbeeck, J. Probing the Symmetry of the Potential of Localized Surface Pplasmon Resonances with Phase-Shaped Electron Beams. Nat. Commun. 2017, 8, 14999.

(12) Krehl, J.; Guzzinati, G.; Schultz, J.; Potapov, P.; Pohl, D.; Martin, J.; Verbeeck, J.; Fery, A.; Büchner, B.; Lubk, A. Spectral Field Mmapping in Plasmonic Nanostructures with Nanometer Resolution. Nat. Commun. 2018, 9, 4207.

(13) García de Abajo, F. J.; A. G. Pattantyus-Abraham,; Zabala, N.; Rivacoba, A.; Wolf, M. O.; Echenique, P. M. Cherenkov Effect as a Probe of Photonic Nanostructures. Phys. Rev. Lett. 2003, 91, 143902.

(14) Peng, S.; Schilder, N. J.; Ni, X.; van de Groep, J.; Brongersma, M. L.; Alù, A.; Khanikaev, A. B.; Atwater, H. A.; Polman, A. Probing the Band Structure of Topological Silicon Photonic Lattices in the Visible Spectrum. Phys. Rev. Lett. 2019, 122, 117401.

(15) Wang, K.; Dahan, R.; Shentcis, M.; Kauffmann, Y.; Tsesses, S.; ; Kaminer, I. Coherent Interaction between Free Electrons and Cavity Photons. 2019, 1908.06206. arXiv. https://arxiv.org/abs/1908.06206 (accessed April 16, 2020).

(16) Liu, F.; Xiao, L.; Ye, Y.; Wang, M.; Cui, K.; Feng, X.; Zhang, W.; Huang, Y. Integrated Cherenkov Radiation Emitter Eliminating the Electron Velocity Threshold. Nat. Photon. 2017, 11, 289-292.

(17) Tsesses, S.; Bartal, G.; Kaminer, I. Light Generation via Quantum Interaction of Electrons with Periodic Nanostructures. Phys. Rev. A 2017, 95, 013832.

(18) Barwick, B.; Flannigan, D. J.; Zewail, A. H. Photon-Induced Near-Field Electron Microscopy. Nature 2009, 462, 902-906. 
(19) Feist, A.; Echternkamp, K. E.; Schauss, J.; Yalunin, S. V.; Schäfer, S.; Ropers, C. Quantum Coherent Optical Phase Modulation in an Ultrafast Transmission Electron Microscope. Nature 2015, 521, 200-203.

(20) Vanacore, G. M.; Madan, I.; Berruto, G.; Wang, K.; Pomarico, E.; Lamb, R. J.; McGrouther, D.; Kaminer, I.; Barwick, B.; García de Abajo, F. J.; Carbone, F. Attosecond Coherent Control of Free-Electron Wave Functions Using Semi-Infinite Light Fields. Nat. Commun. 2018, 9, 2694.

(21) García de Abajo, F. J. Multiple Excitation of Confined Graphene Plasmons by Single Free Electrons. ACS Nano 2013, 7, 11409-11419.

(22) Cox, J. D.; García de Abajo, F. J. Single-Plasmon Thermo-Optical Switching in Graphene. Acc. Chem. Res. 2019, 52, 2536-2547.

(23) Mikhailov, S. A.; Ziegler, K. Nonlinear Electromagnetic Response of Graphene: Frequency Multiplication and the Self-Consistent-Field Effects. J. Phys. Condens. Matter 2008, 20, 384204 .

(24) Cheng, J. L.; Vermeulen, N.; Sipe, J. E. Third-Order Nonlinearity of Graphene: Effects of Phenomenological Relaxation and Finite Temperature. Phys. Rev. B 2015, 91, 235320 .

(25) Mikhailov, S. A. Quantum Theory of the Third-Order Nonlinear Electrodynamic Effects of Graphene. Phys. Rev. B 2016, 93, 085403.

(26) Ju, L.; Geng, B.; Horng, J.; Girit, C.; Martin, M.; Hao, Z.; Bechtel, H. A.; Liang, X.; Zettl, A.; Shen, Y. R.; Wang, F. Graphene Plasmonics for Tunable Terahertz Metamaterials. Nat. Nanotech. 2011, 6, 630-634.

(27) Fei, Z.; Andreev, G. O.; Bao, W.; Zhang, L. M.; McLeod, A. S.; Wang, C.; Stewart, M. K.; Zhao, Z.; Dominguez, G.; Thiemens, M.; Fogler, M. M.; Tauber, M. J.; 
Castro-Neto, A. H.; Lau, C. N.; Keilmann, F.; Basov, D. N. Infrared Nanoscopy of Dirac Plasmons at the Graphene-SiO 2 Interface. Nano Lett. 2011, 11, 4701-4705.

(28) Shin, S. Y.; Kim, N. D.; Kim, J. G.; Kim, K. S.; Noh, D. Y.; Kim, K. S.; Chung, J. W. Control of the $\pi$ Plasmon in a Single Layer Graphene by Charge Doping. Appl. Phys. Lett. 2011, 99, 082110.

(29) Fei, Z.; Rodin, A. S.; Andreev, G. O.; Bao, W.; McLeod, A. S.; Wagner, M.; Zhang, L. M.; Zhao, Z.; Thiemens, M.; Dominguez, G.; Fogler, M. M.; Neto, A. H. C.; Lau, C. N.; Keilmann, F.; Basov, D. N. Gate-Tuning of Graphene Plasmons Revealed by Infrared Nano-Imaging. Nature 2012, 487, 82-85.

(30) Chen, J.; Badioli, M.; Alonso-González, P.; Thongrattanasiri, S.; Huth, F.; Osmond, J.; Spasenović, M.; Centeno, A.; Pesquera, A.; Godignon, P.; Zurutuza Elorza, A.; Camara, N.; García de Abajo, F. J.; Hillenbrand, R.; Koppens, F. H. L. Optical NanoImaging of Gate-Tunable Graphene Plasmons. Nature 2012, 487, 77-81.

(31) Yan, H.; Li, X.; Chandra, B.; Tulevski, G.; Wu, Y.; Freitag, M.; Zhu, W.; Avouris, P.; Xia, F. Tunable Infrared Plasmonic Devices Using Graphene/Insulator Stacks. Nat. Nanotech. 2012, 7, 330-334.

(32) Brar, V. W.; Jang, M. S.; Sherrott, M.; Lopez, J. J.; Atwater, H. A. Highly Confined Tunable Mid-Infrared Plasmonics in Graphene Nanoresonators. Nano Lett. 2013, 13, $2541-2547$.

(33) Yan, H.; Low, T.; Zhu, W.; Wu, Y.; Freitag, M.; Li, X.; Guinea, F.; Avouris, P.; Xia, F. Damping Pathways of Mid-Infrared Plasmons in Graphene Nanostructures. Nat. Photon. 2013, 7, 394-399.

(34) Cox, J. D.; García de Abajo, F. J. Electrically Tunable Nonlinear Plasmonics in Graphene Nanoislands. Nat. Commun. 2014, 5, 5725. 
(35) Cox, J. D.; Yu, R.; García de Abajo, F. J. Analytical Description of the Nonlinear Plasmonic Response in Nanographene. Phys. Rev. B 2017, 96, 045442.

(36) Smith, D. D.; Yoon, Y.; Boyd, R. W.; Campbell, J. K.; Baker, L. A.; Crooks, R. M.; George, M. $z$-Scan Measurement of the Nonlinear Absorption of a Thin Gold Film. J. Appl. Phys. 1999, 86, 6200-6205.

(37) Castro Neto, A. H.; Guinea, F.; Peres, N. M. R.; Novoselov, K. S.; Geim, A. K. The Electronic Properties of Graphene. Rev. Mod. Phys. 2009, 81, 109-162.

(38) Bauer, E. Surface Microscopy with Low Energy Electrons; Springer: New York, 2014.

(39) Tromp, R. In Handbook of Microscopy; Hawkes, P. W., Spence, J. C. H., Eds.; Springer, 2019; pp 565-604.

(40) Ye, R.; Xiang, C.; Lin, J.; Peng, Z.; Huang, K.; Yan, Z.; Cook, N. P.; Samuel, E. L.; Hwang, C.-C.; Ruan, G.; Ceriotti, G.; Raji, A.-R. O.; Martí, A. A.; Tour, J. M. Coal as an Abundant Source of Graphene Quantum Dots. Nat. Commun. 2013, 4, 2943.

(41) Pendry, J. B. In Determination of Surface Structure by LEED; Marcus, P. M., Jona, F., Eds.; Plenum Press: New York, 1984; p 3.

(42) Schefold, J.; Meuret, S.; Schilder, N.; Coenen, T.; Agrawal, H.; Garnett, E. C.; Polman, A. Spatial Resolution of Coherent Cathodoluminescence Super-Resolution Microscopy. ACS Photonics 2019, 4, 1067-1072.

(43) Parilis, E. S.; Kishinevsky, L. M.; Turaev, N. Y.; Baklitzky, B. E.; Umarov, F. F.; Verleger, V. K.; Nizhnaya, S. L.; Bitensky, I. S. Atomic Collisions on Solid Surfaces; Noth-Holland: Amsterdam, 1993.

(44) Konečná, A.; Di Giulio, V.; Mkhitaryan, V.; Ropers, C.; García de Abajo, F. J. Nanoscale Nonlinear Spectroscopy with Electron Beams. ACS Photonics 2020, DOI: 10.1021/acsphotonics.0c00326.. 
(45) Thongrattanasiri, S.; Manjavacas, A.; García de Abajo, F. J. Quantum Finite-Size Effects in Graphene Plasmons. ACS Nano 2012, 6, 1766-1775.

(46) Hedin, L.; Lundqvist, S. In Solid State Physics; Frederick Seitz, D. T., Ehrenreich, H., Eds.; Solid State Physics; Academic Press, 1970; Vol. 23; pp 1-181. 